\title{
Factors Associated with COVID-19 Vaccine Booster Hesitancy: a Retrospective Cohort Study, Fukushima Vaccination Commu- nity Survey
}

\author{
Makoto Yoshida, ${ }^{1}$ Yurie Kobashi, M.D., MPH, ${ }^{2,3}$ Takeshi Kawamura, Ph.D., ${ }^{4,5}$ Yuzo Shimazu, M.D., ${ }^{2}$ Yoshitaka \\ Nishikawa, M.D, Ph.D., ${ }^{3}$ Fumiya Omata, M.D., ${ }^{3}$ Tianchen Zhao, ${ }^{2}$ Chika Yamamoto, R.N., ${ }^{6}$ Yudai Kaneko, M.Eng. ${ }^{5,7}$ \\ Aya Nakayama, B.S., ${ }^{4}$ Morihito Takita, M.D., Ph.D., ${ }^{2,8}$ Naomi Ito, ${ }^{2}$ Moe Kawashima, ${ }^{2}$ Sota Sugiura, ${ }^{8}$ Kenji Shibuya, \\ M.D., DrPH, ${ }^{9,10}$ Shingo Iwami, Ph.D., ${ }^{11}$ Kwangsu Kim, Ph.D., ${ }^{11}$ Shoya Iwanami, Ph.D., ${ }^{11}$ Tatsuhiko Kodama, M.D., \\ Ph.D., ${ }^{5}$ Masaharu Tsubokura, M.D., Ph.D. ${ }^{2,3,8,12^{*}}$
}

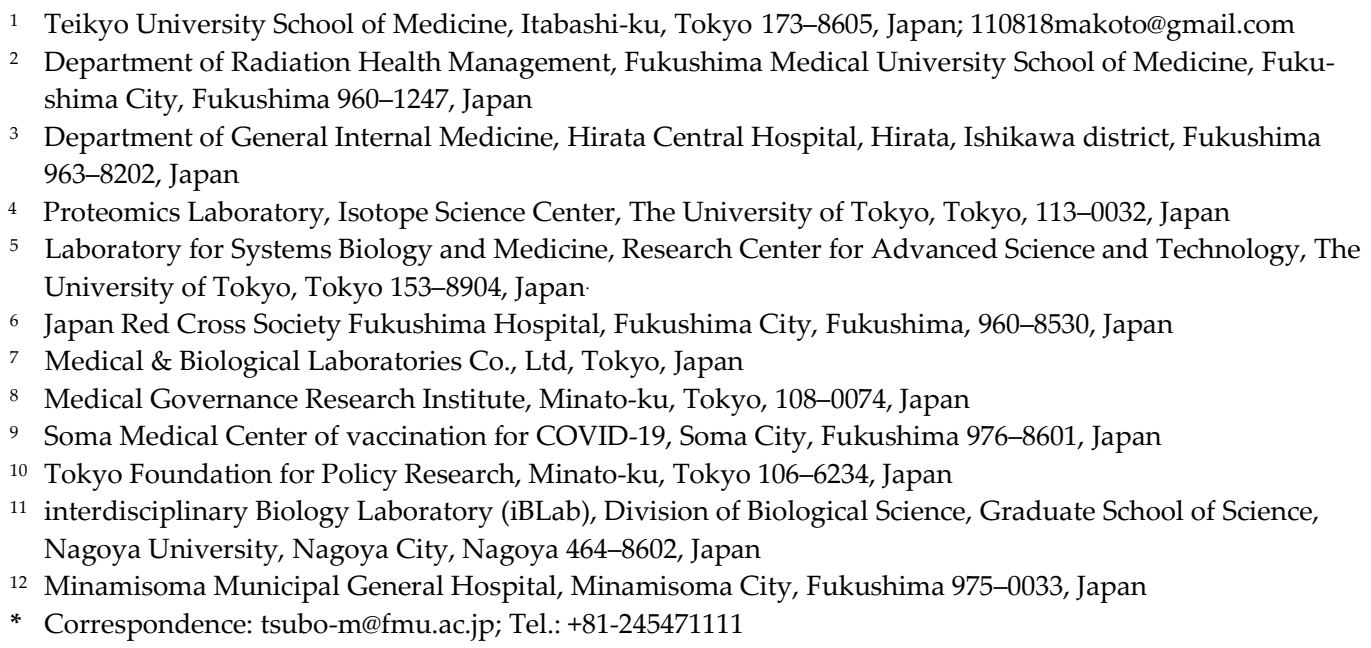

\begin{abstract}
This was a retrospective cohort study, which aimed to investigate the factors associated with hesitancy to receive the third dose of coronavirus disease 2019 (COVID-19) vaccine. A paperbased questionnaire survey was administered to all participants. Accordingly, the study included participants who provided answer in the questionnaire whether they have an intent to receive the third dose of vaccine. Data on sex, age, area of residence, adverse reactions after the second vaccination, whether the third vaccination was desired, and reasons to accept or hesitate booster vaccination were retrieved. Among the 2439 participants with mean $( \pm \mathrm{SD})$ age of $52.6 \pm 18.9$ years, and median IgG-S antibody titer of $324.9(\mathrm{AU} / \mathrm{mL}), 97.9 \%$ of participants indicated their intent to accept a third vaccination dose. The logistic regression revealed that younger age (OR $=0.98 ; 95 \%$ CI: 0.96 1.00) and higher antibody level (OR=2.52; 95\% CI: 1.27-4.99) are positively associated with the third vaccine hesitancy. The efficacy of the COVID-19 vaccine and concerns about adverse reactions had significant impact on the third vaccination behavior. A rapid increase in the booster dose rate is needed to control the pandemic, and specific approaches should be taken in these groups that are likely to hesitate the third vaccine, subsequently increasing booster contact rate.
\end{abstract}

Keywords: antibody; BNT162b2; coronavirus disease 2019; severe acute respiratory syndrome coronavirus 2; vaccine hesitancy; vaccine booster

\section{Introduction}

The coronavirus disease 2019 (COVID-19) was first confirmed in China in December 2019, causing pneumonia and other respiratory diseases and rapidly spreading worldwide with a cumulative total of 400,846,690 confirmed cases and 5,764,834 deaths as of 
February 9, 2022 [1]. Given its high prevalence, an effective vaccine will play a critical role in preventing the spread of COVID-19 [2]. Many efforts have been made to develop vaccines, including the BNT162b2, mRNA-1273 Moderna vaccine, Janssen Ad26.COV2.S, and Sinopharm inactivated virus vaccines, which were approved by the World Health Organization [3]. However, vaccine hesitancy has been observed worldwide and is a major obstacle in controlling the COVID-19 pandemic [4-7]. In addition, there are concerns that the neutralizing activity of the vaccine may decrease over time, even in individuals who received two doses of the vaccine. It was reported that six months after the administration of the second dose of the BNT162b2 vaccine, the humoral response was substantially reduced [8]. The Centers for Disease Control and Prevention has recommended a third COVID-19 vaccine dose in immunocompromised individuals [9]. As described above, reducing the number of unvaccinated individuals and minimizing vaccine hesitancy to facilitate more people receiving a third dose, is an extremely important measure in controlling the COVID-19 pandemic.

Previous studies examined the factors associated with vaccine hesitancy. Ethnicity, work status, religion, politics, sex, age, education, and income have been reported to be factors associated with COVID-19 vaccine hesitancy [10,11]. However, information on the factors affecting COVID-19 booster vaccine hesitancy among fully-vaccinated individuals is limited.

Fukushima Prefecture experienced a surge in COVID-19 cases in August 2021 and January 2022, wherein a total of 17,327 cases, equivalent to 194 cases per 100,000 people, were reported by February 9, 2022 [12]. BNT162b2 vaccinations were offered beginning March 2021 to healthcare workers, older adults, people with comorbidities, and the general population. A total of $81.7 \%$ of the population aged $\geq 12$ years who were eligible for vaccination received second doses by February 2, 2022. The third vaccination dose was offered starting December 1, 2021, to healthcare workers and residents of geriatric facilities who had been vaccinated with BNT162b2 or mRNA-1273 vaccine for at least six months after the second dose, and older adults aged 65 years or older who had been vaccinated for at least eight months. In Japan, 3.96\% of the population received a third dose by February 3, 2022, which is the lowest among the OECD member countries. In Fukushima Prefecture, $5.1 \%$ of the total population completed the third dose as of February 3, 2022 [13]. In addition, certain disaster-affected populations in Fukushima have been continuously subjected to COVID-19 antibody titers monitoring to develop infection prevention measures accordingly [14,15]. This area has an advantage in that the residents are aware of the level of their own liquid immunity after vaccination and how it has evolved, allowing assessment on how this affects their third vaccination behavior.

The purpose of this study was to investigate factors associated with the third dose of COVID-19 vaccine hesitancy to increase the booster contact rate required to control the COVID-19 pandemic. This study was supported by the Japan Agency for Medical Research and Development and was conducted as part of the FVCS, a study to evaluate antibody titer dynamics after the second dose of the BNT162b2 vaccine in rural communities in Japan.

\section{Materials and Methods}

\section{Study Design and Population}

This was a retrospective, cohort study. The participants included healthcare workers, frontline workers, administrative officers, general residents, and residents of long-term care facilities. Hospital groups and municipalities in Kenchu District and Soso District in Fukushima Prefecture cooperated to recruit participants and perform blood sampling between September 8 and October 8, 2021. Participants were mainly recruited from rural Fukushima Prefecture, including Ishikawa gun, Soma City, and Minamisoma City. A total of 1,432, 500, and 594 participants were recruited from Ishikawa gun, Soma City, and Minamisoma City, respectively. 
In December 2021, a paper-based questionnaire survey inquiring information regarding sex, age, area of residence, adverse reactions after the second vaccination, whether the third vaccination was desired, and reasons for wanting or not wanting the vaccination was distributed to all participants. Subsequently, the eligibility criterion for the study participants is that they provided answer in the questionnaire whether they have an intent to receive the third dose of vaccine. Children aged $\geq 12$ years were allowed to receive the COVID-19 vaccination; thus, we included 58 participants aged 12-19 years.

\section{Questionnaire Survey on Whether or Not Booster Vaccination is Accepted and Why}

To reflect the opinions of the residents, we included a question in the questionnaire whether they wanted to receive the third vaccination dose and why, with input from the medical staff, local government staff, and researchers involved in the vaccination process. The items in the questionnaire inquiring for reasons can be answered in multiple ways and were designed such that the reasons for acceptance corresponded to the reasons for hesitancy. A paper on vaccine hesitancy was used as a reference in preparing the questionnaire survey $[10,11]$.

\section{Serological Assay}

Blood sampling was conducted between September 8 and October 8, 2021, at each facility in the rural Fukushima Prefecture. Centrifugal separation of blood samples was performed at each facility and serum samples were sent to Tokyo University. The levels of immunoglobulin $\mathrm{G}$ (IgG) antibodies against the severe acute respiratory syndrome coronavirus 2 (SARS-CoV-2) spike (S1) protein were measured at Tokyo University. Serological assays were performed using the CLIA assay with iFlash 3000 (YHLO Biotech, Shenzhen, China) and iFlash-2019-nCoV series (YHLO Biotech) as reagents. The cut-off value for IgG antibody against $S 1$ protein was ten arbitrary units per milliliter (AU/mL), which was the standard cut-off value. The testing process complied with the official guidelines [16,17]. The results of the serological assay were informed to the participants in November 2021.

\section{Statistical Analysis}

We compared the characteristics of participants according to their willingness to receive a third COVID-19 vaccine dose. We conducted a chi-square test for sex, municipality, and adverse reactions, a t-test for age, and a Wilcoxon rank-sum test for IgG antibody titer. The reasons to accept or hesitate the third vaccination dose were tabulated separately. A logistic regression was used to assess the relationship between third vaccine hesitancy and age, sex, number of adverse reactions after the second vaccination, IgG-S antibody titer higher or lower than the median (324.85 AU/ml), and place of residence. A logistic regression was used to assess the relationship between third vaccine hesitancy and age, sex, fever above $37.5^{\circ} \mathrm{C}$, fatigue, headache, joint pain, nausea, IgG-S antibody titer above and below the median $(324.85 \mathrm{AU} / \mathrm{ml})$ after the second vaccination and place of residence. We considered $\mathrm{p}$ values of 0.05 or less to be significant. The statistical software STATA IC (version 15; Lightstone, Texas, USA) was used for all analyses.

\section{Results}

Among the 2439 participants with a mean $( \pm \mathrm{SD})$ age of $52.6 \pm 18.9$ years, median IgG$\mathrm{S}$ antibody titer of $324.9(\mathrm{AU} / \mathrm{mL})$, and of whom $41.7 \%$ were male, $97.9 \%$ indicated their intent to accept the third vaccination dose. The mean age was significantly lower $(p=0.001)$, while the median IgG-S score was significantly higher in the vaccine-hesitancy group $(p<0.001)$. The frequency of nausea as an adverse reaction after the second vaccination was also significantly higher in the vaccine-hesitancy group $(p<0.001)$ (Table 1$)$. 
Table 1. Characteristics of participants by willingness to avail of the third COVID-19 vaccine dose $n$ (\%) $(\mathrm{N}=2439)$

\begin{tabular}{|c|c|c|c|}
\hline & Accept Vaccine & Hesitate Vaccine & Total \\
\hline $\operatorname{Age}^{* *}($ mean $[S D])$ & 52.8 [18.9] & $44.0[19.0]$ & $52.6[19.3]$ \\
\hline \multicolumn{4}{|l|}{ Sex } \\
\hline Male & $998(41.8)$ & $20(38.5)$ & 1018 (41.7) \\
\hline $\begin{array}{l}\text { IgG antibody } \operatorname{titer}^{* * *} \text { (median }\left(25^{\text {th }}\right. \\
\left.\left.\qquad 75^{\text {th }}\right)\right)\end{array}$ & $\begin{array}{l}319.6(171.7- \\
571.3)\end{array}$ & $\begin{array}{c}549.9(446.0- \\
724.1)\end{array}$ & $\begin{array}{c}324.9(173.2- \\
576.3)\end{array}$ \\
\hline \multicolumn{4}{|l|}{ Municipality* } \\
\hline Hirata & $1343(56.3)$ & $37(71.2)$ & $1380(56.6)$ \\
\hline Soma & $464(19.4)$ & $5(9.6)$ & $469(19.2)$ \\
\hline Minamisoma & $580(24.3)$ & $10(19.2)$ & $590(24.2)$ \\
\hline \multicolumn{4}{|l|}{ Adverse reaction } \\
\hline Local Pain & $1,358(57.0)$ & $24(46.2)$ & $1,382(56.8)$ \\
\hline Over $37.5^{\circ} \mathrm{C}$ fever $*$ & $676(28.4)$ & $21(40.4)$ & $697(28.7)$ \\
\hline Fatigue & $1,195(50.2)$ & $28(53.9)$ & $1223(50.3)$ \\
\hline Headache $^{* *}$ & $646(27.1)$ & $22(42.3)$ & $668(27.4)$ \\
\hline Joint pain & $728(30.6)$ & $18(34.6)$ & $746(30.7)$ \\
\hline Diarrhea & $53(2.2)$ & $2(3.9)$ & $55(2.3)$ \\
\hline Nausea $^{* * *}$ & $87(3.7)$ & 87 (17.3) & $96(3.9)$ \\
\hline Dizziness & $102(4.3)$ & $3(5.8)$ & $105(4.3)$ \\
\hline
\end{tabular}

We conducted a chi-square test for sex, municipality, and adverse reactions, a t-test for age, and a Wilcoxon rank-sum test for IgG antibody titer.

***: p-value $<0.001$

**: p-value $<0.05$

*: p-value $<0.1$

The main reasons for accepting the third vaccination were that it is necessary for infection control (81.8\%), vaccines are highly effective $(47.3 \%)$, and adverse reactions are not a major concern $(27.1 \%)$. The main reasons to hesitate the third vaccination were worried about adverse reactions (57.7\%), two doses of vaccine are sufficient $(25.0 \%)$, and efficacy is unknown (19.2\%) (Table 2). Concerns about adverse reactions and efficacy of the vaccine were the top reasons to hesitate a third vaccination. In cases where the respondents were not concerned about efficacy or adverse reactions, these factors were the top reasons for accepting the third vaccination.

Table 2. Reasons to accept or hesitate a third COVID-19 vaccine dose $(\mathrm{N}=2439)$

$$
\mathrm{n}(\%)
$$

Reasons to accept the third vaccination $(n=2387)$

Necessary for infection control

Vaccines are highly effective 
Adverse reactions are not a major concern

$647(27.1)$

The second vaccination is not effective enough

$494(20.7)$

Considering the number of people infected with the COVID-19

$325(13.6)$

$$
\text { Vaccine is safe }
$$

Access to vaccination sites is good

300 (12.6)

Low antibody levels

$280(11.7)$

I have a chronic disease

$181(7.6)$

No work shift

Reasons to hesitate the third vaccination $(n=52)$

Worried about adverse reactions

Two doses of vaccine are sufficient

Efficacy is unknown

Worried about long term effects

Too much trouble

The number of people infected with COVID-19 is small.

Antibody titers are high

Other methods of infection control are sufficient

I can't take a day off work or school.

Results of the logistic regression revealed that younger age (OR=0.98; 95\% CI: 0.96$1.00)$ and higher antibody level (OR=2.52; 95\% CI: 1.27-4.99) are predominantly positively associated with the third vaccine hesitancy. In contrast, sex and the number of adverse reactions at the second vaccination were not significantly associated with third vaccine hesitancy (Table 3). Furthermore, the results of the multilevel model analysis showed that younger age and experience of adverse reactions to nausea are significantly associated with third vaccine hesitancy $(\mathrm{p}<0.001)$ (Supplementary Table 1).

Table 3. Logistic regression analysis to identify the variables which effected on vaccine hesitancy

\begin{tabular}{cccc}
\hline & B (se) & OR (95\% CI) & p-value \\
\hline Age & -0.021 & $0.98(0.96-1.00)$ & 0.030 \\
Sex (base: male) & 0.066 & $1.07(0.60-1.92)$ & 0.82 \\
Number of whole-body adverse reaction & 0.049 & $1.05(0.87-1.27)$ & 0.61 \\
Municipality (base: Hirata) & 0.920 & $2.52(1.27-4.99)$ & 0.008 \\
$\quad$ Soma & & & 0.031 \\
Minamisoma & -1.060 & $0.35(0.13-0.91)$ & 0.113 \\
\hline
\end{tabular}

Whole-body adverse reactions include over $37.5^{\circ} \mathrm{C}$ fever, fatigue, headache, joint pain, diarrhea, nausea, and dizziness.

\section{Discussion}


Reducing vaccine hesitancy is important in controlling the COVID-19 pandemic. In this study, we conducted a retrospective cohort study by distributing questionnaires in December 2021 to populations with measured IgG-S antibody titers for COVID-19 between September 8 and October 8, 2021, in rural areas of Japan to investigate the factors associated with the third vaccine hesitancy.

In this study, we found that people who acquired relatively high liquid immunity after two doses of vaccination were associated with a third vaccine hesitancy. Younger age (OR=0.98; 95\% CI: 0.96-1.00) and higher previous antibody titer than the median $(\mathrm{OR}=2.52 ; 95 \% \mathrm{CI}: 1.27-4.99)$ were positively associated with vaccine hesitancy. This result is consistent with the previous reports that younger individuals are less likely to accept vaccines [5,18-21]. Since antibody levels decrease over time [8], it is not guaranteed that the third vaccination is unnecessary when the antibody level is higher than the median. Thus, it is essential to provide information about the importance of a third dose and discuss how to interpret the results of antibody levels.

Approximately $97.9 \%$ of FVCS participants who had their own antibody titers measured accepted a third vaccination. The estimated global acceptance rate of the COVID-19 vaccine was reported to be at $73.2 \%$ [22], which is lower than our results. It is also suggested that education and policy-based interventions for vaccination should be implemented [10,22-25]. Continuous antibody testing, careful explanation of test results on paper, and information sharing through meetings among medical staff may have contributed to the high vaccination acceptance rate.

The efficacy of the COVID-19 vaccine and concerns about adverse reactions had a significant impact on the reasons for the third vaccination behavior. Previous studies have shown that vaccine efficacy, safety, and lack of concern regarding adverse reactions increase vaccine acceptance $[10,22,26-33]$. It is important to continue to consider these factors when designing communication about vaccines.

A limitation of this study is that the participants were recruited in the disaster-affected area using a unique network developed with more than 10 years of disaster recovery through a multi-sectoral partnership. These participants are interested in vaccines and health issues, and voluntarily signed-up to participate in the FVCS. This may introduced a sampling bias and may not be conclusive of other populations. In addition, it was not possible to eliminate potential confounding factors such as media preferences, education, and income.

\section{Conclusions}

In this study, we found that younger age groups, and participants with antibody levels higher than the median were associated with the third vaccine hesitancy. A rapid increase in the booster dose rate is needed to control the virus, and specific approaches should be taken in these groups that are likely to hesitate the third vaccine. Further research on factors associated with the third vaccine hesitancy should also be explored.

Supplementary Materials: The following supporting information can be downloaded at: www.mdpi.com/xxx/s1, Table S1: A logistic regression to identify variables associated with vaccine hesitancy.

Author Contributions: Conception of the work, M.Y., Y.K, M.T.; data collection, all authors; investigation, Y.K., M.T.; data analysis and interpretation, MY, Y.K, M.T.; data curation, M.Y., Y.K.; drafting of the article, M.Y., Y.K., Y.N., F.O., M.T.; critical revision of the article, all authors. All authors have read and agreed to the published version of the manuscript.

Funding: This research was supported by AMED under Grant Number JP21nf0101638 and by Medical \& Biological Laboratories Co., Ltd. and Shenzhen YHLO Biotech Co., Ltd., the distributor and manufacturer of the antibody measurement system (iFlash 3000). This research was also supported by grants from Kowa Co. and the Research Center for Advanced Science and Technology at the University of Tokyo.

Institutional Review Board Statement: This study was approved by the ethics committees of Hirata Central Hospital (number 2021-0611-1) and Fukushima Medical University (number 2021-116). This 
study was conducted in accordance with the Code of Ethics of the World Medical Association (Declaration of Helsinki).

Informed Consent Statement: Informed consent was obtained from all subjects involved in the study.

Data Availability Statement: Not applicable

Acknowledgments: We would like to thank all the staff from Fukushima Medical University, Seireikai health care group, Hirata Village office, Soma City office, Soma Central Hospital, Soma General Hospital, Minamisoma City office, Minamisoma City Medical Association, Minamisoma Municipal General Hospital, Shindo Clinic and Medical Governance Institute, who contributed significantly to the accomplishment of this research, especially Ms. Yuka Harada, Ms. Serina Noji, Mr. Masahiko Nihei, Mr. Hideo Sato, Ms. Rie Yanai, Ms. Yasuko Suzuki, Ms. Keiko Abe, Dr. Hidekiyo Tachiya, Mr. Kouki Nakatsuka, Dr. Ryuzaburo Shineha, Ms. Miki Sato, Dr. Masahiko Sato, Mr. Naoharu Tadano, Mr. Kazuo Momma, Mr. Shu-ichi Mori, Ms. Saori Yoshisato, Ms. Katsuko Onoda, Mr. Satoshi Kowata, Mr. Masatsugu Tanaki, Dr. Tomoyoshi Oikawa, Dr. Joji Shindo, Ms. Xujin Zhu, and Ms. Tomoyo Nishimura. We would like to thank Editage (www.editage.com) for English language editing.

Conflicts of Interest: Kobashi and Tsubokura received a research grant from the Pfizer Health Research Foundation for research not associated with this study.

\section{References}

1. Medicine, J.H.U.o. Coronavirus Resource Center. Available online: https://coronavirus.jhu.edu/map.html (accessed on February 9).

2. Lin, C.; Tu, P.; Beitsch, L.M. Confidence and Receptivity for COVID-19 Vaccines: A Rapid Systematic Review. Vaccines 2020, 9, doi:10.3390/vaccines9010016.

3. COVID-19 vaccines. In Drugs and Lactation Database (LactMed); National Library of Medicine (US): Bethesda (MD), 2006.

4. Sallam, M. COVID-19 Vaccine Hesitancy Worldwide: A Concise Systematic Review of Vaccine Acceptance Rates. Vaccines 2021, 9, doi:10.3390/vaccines9020160.

5. Lazarus, J.V.; Ratzan, S.C.; Palayew, A.; Gostin, L.O.; Larson, H.J.; Rabin, K.; Kimball, S.; El-Mohandes, A. Author Correction: A global survey of potential acceptance of a COVID-19 vaccine. Nature medicine 2021, 27, 354, doi:10.1038/s41591-020-012260 .

6. Organization, W.H. Ten threats to global health 2019. Available online: https://www.who.int/news-room/spotlight/tenthreats-to-global-health-in-2019 (accessed on February 9).

7. Attitudes Toward a Potential SARS-CoV-2 Vaccine. Annals of Internal Medicine 2020, 173, 964-973, doi:10.7326/m20-3569 \%m 32886525 .

8. Levin, E.G.; Lustig, Y.; Cohen, C.; Fluss, R.; Indenbaum, V.; Amit, S.; Doolman, R.; Asraf, K.; Mendelson, E.; Ziv, A.; et al. Waning Immune Humoral Response to BNT162b2 Covid-19 Vaccine over 6 Months. New England Journal of Medicine 2021, 385, e84, doi:10.1056/NEJMoa2114583.

9. Shekhar, R.; Garg, I.; Pal, S.; Kottewar, S.; Sheikh, A.B. COVID-19 Vaccine Booster: To Boost or Not to Boost. Infectious disease reports 2021, 13, 924-929, doi:10.3390/idr13040084.

10. Joshi, A.; Kaur, M.; Kaur, R.; Grover, A.; Nash, D.; El-Mohandes, A. Predictors of COVID-19 Vaccine Acceptance, Intention, and Hesitancy: A Scoping Review. Frontiers in public health 2021, 9, 698111, doi:10.3389/fpubh.2021.698111.

11. Wang, Y.; Liu, Y. Multilevel determinants of COVID-19 vaccination hesitancy in the United States: a rapid systematic review. Preventive medicine reports 2021, 25, 101673, doi:10.1016/j.pmedr.2021.101673.

12. Corporation, J.B. Novel coronaviruses data for Fukushima Prefecture. Available online: https://www3.nhk.or.jp/news/special/coronavirus/data/pref/fukushima.html (accessed on February 9).

13. Inc., N. COVID-19 Vaccination Status in Japan Charted. Available online: 
https://vdata.nikkei.com/newsgraphics/coronavirus-japan-vaccine-status/ (accessed on February 4).

14. Kobashi, Y.; Nishikawa, Y.; Kawamura, T.; Kodama, T.; Shimazu, Y.; Obara, D.; Zhao, T.; Tsubokura, M. Seroprevalence of SARS-CoV-2 antibodies among hospital staff in rural Central Fukushima, Japan: A historical cohort study. International immunopharmacology 2021, 98, 107884, doi:https://doi.org/10.1016/j.intimp.2021.107884.

15. Kobashi, Y.; Shimazu, Y.; Nishikawa, Y.; Kawamura, T.; Kodama, T.; Obara, D.; Tsubokura, M. The difference between IgM and IgG antibody prevalence in different serological assays for COVID-19; lessons from the examination of healthcare workers. International immunopharmacology 2021, 92, 107360, doi:https://doi.org/10.1016/j.intimp.2020.107360.

16. Analuzer, I. iFlash-SARS-CoV-2 IgG-S. 2021.

17. Analuzer, I. iFlash-2019-nCoV NAb. 2021, 4.

18. Bish, A.; Yardley, L.; Nicoll, A.; Michie, S. Factors associated with uptake of vaccination against pandemic influenza: A systematic review. Vaccine 2011, 29, 6472-6484, doi:https://doi.org/10.1016/j.vaccine.2011.06.107.

19. Gagneux-Brunon, A.; Detoc, M.; Bruel, S.; Tardy, B.; Rozaire, O.; Frappe, P.; Botelho-Nevers, E. Intention to get vaccinations against COVID-19 in French healthcare workers during the first pandemic wave: a cross-sectional survey. The Journal of hospital infection 2021, 108, 168-173, doi:10.1016/j.jhin.2020.11.020.

20. Al-Mohaithef, M.; Padhi, B.K. Determinants of COVID-19 Vaccine Acceptance in Saudi Arabia: A Web-Based National Survey. Journal of multidisciplinary healthcare 2020, 13, 1657-1663, doi:10.2147/jmdh.S276771.

21. Malik, A.A.; McFadden, S.M.; Elharake, J.; Omer, S.B. Determinants of COVID-19 vaccine acceptance in the US. EClinicalMedicine 2020, 26, 100495, doi:10.1016/j.eclinm.2020.100495.

Wang, Q.; Yang, L.; Jin, H.; Lin, L. Vaccination against COVID-19: A systematic review and meta-analysis of acceptability and its predictors. Preventive Medicine 2021, 150, 106694, doi:https://doi.org/10.1016/j.ypmed.2021.106694.

Krishnamoorthy, Y.; Kannusamy, S.; Sarveswaran, G.; Majella, M.G.; Sarkar, S.; Narayanan, V. Factors related to vaccine hesitancy during the implementation of Measles-Rubella campaign 2017 in rural Puducherry-A mixed-method study. Journal of family medicine and primary care 2019, 8, 3962-3970, doi:10.4103/jfmpc.jfmpc_790_19.

24. Agrawal, A.; Kolhapure, S.; Di Pasquale, A.; Rai, J.; Mathur, A. Vaccine Hesitancy as a Challenge or Vaccine Confidence as an Opportunity for Childhood Immunisation in India. Infectious diseases and therapy 2020, 9, 421-432, doi:10.1007/s40121-02000302-9.

25. Su, Z.; Wen, J.; Abbas, J.; McDonnell, D.; Cheshmehzangi, A.; Li, X.; Ahmad, J.; Šegalo, S.; Maestro, D.; Cai, Y. A race for a better understanding of COVID-19 vaccine non-adopters. Brain, behavior, \& immunity - health 2020, 9, 100159, doi:10.1016/j.bbih.2020.100159.

26. Wang, J.; Jing, R.; Lai, X.; Zhang, H.; Lyu, Y.; Knoll, M.D.; Fang, H. Acceptance of COVID-19 Vaccination during the COVID19 Pandemic in China. Vaccines 2020, 8, doi:10.3390/vaccines8030482.

Harapan, H.; Wagner, A.L.; Yufika, A.; Winardi, W.; Anwar, S.; Gan, A.K.; Setiawan, A.M.; Rajamoorthy, Y.; Sofyan, H.; Mudatsir, M. Acceptance of a COVID-19 Vaccine in Southeast Asia: A Cross-Sectional Study in Indonesia. Frontiers in public health 2020, 8, 381, doi:10.3389/fpubh.2020.00381.

28. Dror, A.A.; Eisenbach, N.; Taiber, S.; Morozov, N.G.; Mizrachi, M.; Zigron, A.; Srouji, S.; Sela, E. Vaccine hesitancy: the next challenge in the fight against COVID-19. European Journal of Epidemiology 2020, 35, 775-779, doi:10.1007/s10654-020-00671-y.

29. Wang, W.; Wu, Q.; Yang, J.; Dong, K.; Chen, X.; Bai, X.; Chen, X.; Chen, Z.; Viboud, C.; Ajelli, M.; et al. Global, regional, and national estimates of target population sizes for covid-19 vaccination: descriptive study. BMJ 2020, 371, m4704, doi:10.1136/bmj.m4704.

30. Peretti-Watel, P.; Seror, V.; Cortaredona, S.; Launay, O.; Raude, J.; Verger, P.; Fressard, L.; Beck, F.; Legleye, S.; L'Haridon, O.; et al. A future vaccination campaign against COVID-19 at risk of vaccine hesitancy and politicisation. The Lancet Infectious Diseases 2020, 20, 769-770, doi:10.1016/S1473-3099(20)30426-6.

31. Bell, S.; Clarke, R.; Mounier-Jack, S.; Walker, J.L.; Paterson, P. Parents' and guardians' views on the acceptability of a future 
COVID-19 vaccine: A multi-methods study in England. Vaccine 2020, 38, 7789-7798, doi:https://doi.org/10.1016/j.vaccine.2020.10.027.

32. Rhodes, A.; Hoq, M.; Measey, M.-A.; Danchin, M. Intention to vaccinate against COVID-19 in Australia. The Lancet Infectious Diseases 2021, 21, e110, doi:10.1016/S1473-3099(20)30724-6.

33. Kajiwara, S.; Akiyama, N.; Ohta, M. Intentions Regarding COVID-19 Vaccination in Females Aged 15\&ndash;49 Years. Vaccines 2022, 10, doi:10.3390/vaccines10020336. 